Egyptian

Orthodontic Journal

\title{
DISTAL JET VERSUS FIRST CLASS APPLIANCE FOR MAXILLARY FIRST MOLAR DISTALIZATION
}

\author{
MAHER A FOUDA* \\ AHMED A EL-BIALY** \\ YASSER L A MAHMOUD***
}

\section{ABSTRACT}

The present study was conducted to evaluate and compare the effects of the Distal Jet and First Class appliances in distalizing maxillary first molars. The sample consisted of twenty female patients with a mean age of 14.6 years. The patients were divided randomly into two equal groups. In the first group Distal Jet appliances were used to distalize the maxillary first molars. On the other hand, First Class appliances were utilized for molar disalization in the second group. The disalization of the first molars were stopped when Class I molar relations were obtained. For all patients pre and post-distalization lateral cephalometric radiographs were taken. The results revealed that either appliance significantly distalized the maxillary first molars. However, the incisors were significantly tipped labially. Also, in both groups; no significant skeletal changes in the anteroposterior direction were found. The mandible showed significant clock-wise rotation. Regarding the difference between the two groups, the distalizing time of the First Class appliance was significantly shorter than that of the Distal Jet. However, the distal tipping of the maxillary first molars was significantly greater in the First Class group than in the Distal Jet group. The other parameters were not significantly different between the two groups. According to the results of the present study; the following could be concluded; 6oth the Distal Jet and the First Class

\footnotetext{
* Professor of Orthodontics, Faculty of Dentistry, Mansoura University, Egypt.

** Associate Professor Orthodontics, Faculty of Dentistry, Mansoura University, Egypt.

${ }^{* * *}$ Lecturer of Orthodontics, Faculty of Dentistry, Mansoura University, Egypt.
} 
Egyptian

Orthodontic Journal

appliances were effective and reliable methods for maxillary first molar distalization. The First Class appliance distalized the maxillary molars in shorter time than the Distal Jet appliance 6ut with greater distal tipping. Labial tipping of the incisors and clock-wise mandibular rotation occurred with both appliances. $\mathcal{N}$ o significant skeletal changes in the anteroposterior direction found with either appliances.

\section{INTRODUCTION}

Extraction and non-extraction are two opposite treatment strategies. Each has its own advantages. One of the most important advantages of nonextraction treatment is the preservation of sound teeth. ${ }^{1}$ Non extraction treatments could be established by expansion or distalization of the molars. Distalization may be indicated for patients with mild to moderate crowding or patients with maxillary dentoalveolar protrusion or minor skeletal discrepancies. ${ }^{1}$ However; it is not indicated for hyperdivergent patients and those who exhibit significant dental crowding. ${ }^{2,3}$

Non extraction treatment of Class II malocclusions requires distalization of maxillary molars to achieve a Class I relationship. A large number of molar distalizers have been developed, including those compliance and non-compliance appliances.

Extraoral traction with facebow or headgear $\mathrm{J}$ hooks with sliding jig and intraoral removable acrylic appliance with finger springs or Wilson appliance with intermaxillary elastics are examples for compliance appliance. Despite their success in tooth movement, all these modalities have the major disadvantage of heavy dependence on the patient cooperation. ${ }^{4-8}$

Since patient cooperation is the most important key to any treatment success treatment that decrease dependence on patient cooperation may produce more predictable results than those that require cooperation. ${ }^{2}$ In line with this demand a large variety of non compliance "fixed" appliances were introduced. Among those appliances are; Jones jig, ${ }^{9-11}$ Pendulum, ${ }^{12-16}$ repelling magnets, ${ }^{17,} 18$ multidistalizing arch, ${ }^{19 \mathrm{ect}}$.

However, most of those systems produce certain amount of anterior anchorage loss. Also, they tend to produce some distal tipping of the maxillary molars, rather than pure bodily movement. Those limitations introduce inefficiencies of the Class II correction and require a second phase of molar uprighting. ${ }^{2}, 10,14$, and 20

Volume 28 - December 2005 
Carano and Testa (1996) developed and introduced the Distal Jet appliance for molar distalization aiming to overcome the disadvantages of other distalizers. ${ }^{20}$ The distalizing forces were obtained from palatally situated coil springs. They mentioned several advantages for the Distal Jet such as; it is relatively easy to insert, well tolerated, esthetically acceptable and requires no patient cooperation. Studies made to evaluate the effect of the Distal Jet revealed that the appliance is reliable method for maxillary molar distalization since the maxillary molars are distalized with less distal tipping and without the lingual movement that occurs with other distalizers such as the pendulum. However, some anchorage loss in premolars and incisors was found. ${ }^{2,19-21}$

Fortini et al (1999) developed a noncompliance appliance for maxillary molar distalization. ${ }^{22}$ They termed it the First Class appliance. Regarding this appliance, the distalizing forces were exerted from both the buccal and lingual aspects since the appliance incorporated formative screws on the buccal aspects of the teeth in addition to coil springs on the palatal aspects. Studies made to evaluate effects of the First Class appliance concluded that this appliance distalized the maxillary first molars rapidly and effectively. ${ }^{22-24}$

In spite of the several studies that have been made evaluating either the distal Jet or the First Class appliance for maxillary molar distalization no study was found comparing their effects. Therefore, the aim of the present study was to evaluate the effects of the Distal Jet appliance versus the First Class appliance in distalizing the maxillary first molars.

\section{Materials and Methods:}

The sample of the present study consisted of 20 female patients. The mean age of the sample was $14.6(\mathrm{SD} \pm 2.5)$. The subjects were selected according to the following criteria:

- Mild to moderate crowding (3-6mm).

- Class II molar relation.

- Skeletal Class 1.

- No oral habits.

- No previous orthodontic treatment.

The patients were divided randomly into two equal groups. In the first group Distal Jet* appliances were used to distalize the maxillary first molars. On the other hand, First Class* appliances were utilized for molar disalization in the second group. The construction of either distalizer was done according to the manufacturers instructions. The Nance buttons were anchored by supporting

\footnotetext{
* American Orthodontics, USA.

* Leone Company, Italy.
} 
wires to the first premolars in the Distal Jet appliance (Figure 1) and to the second premolars in the first Class appliance (Figure 2).

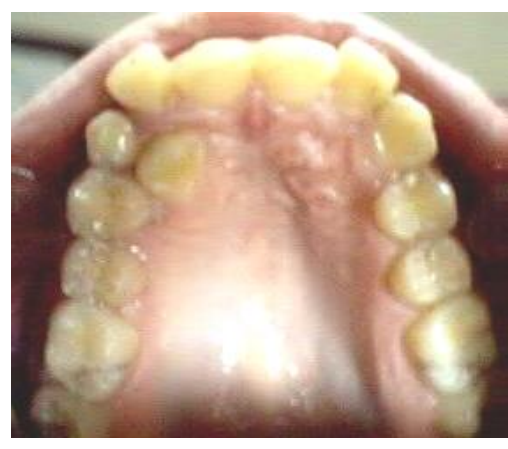

a. Pre-distalization.

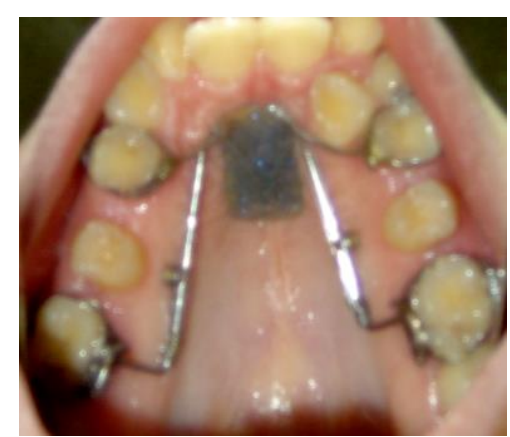

b. Post-distalization.

Figure 1: The Distal Jet appliance.

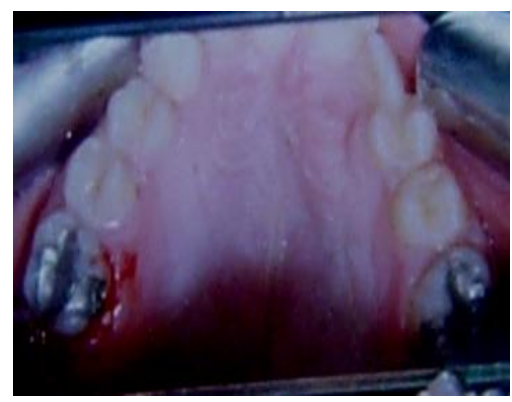

a. Pre-distalization
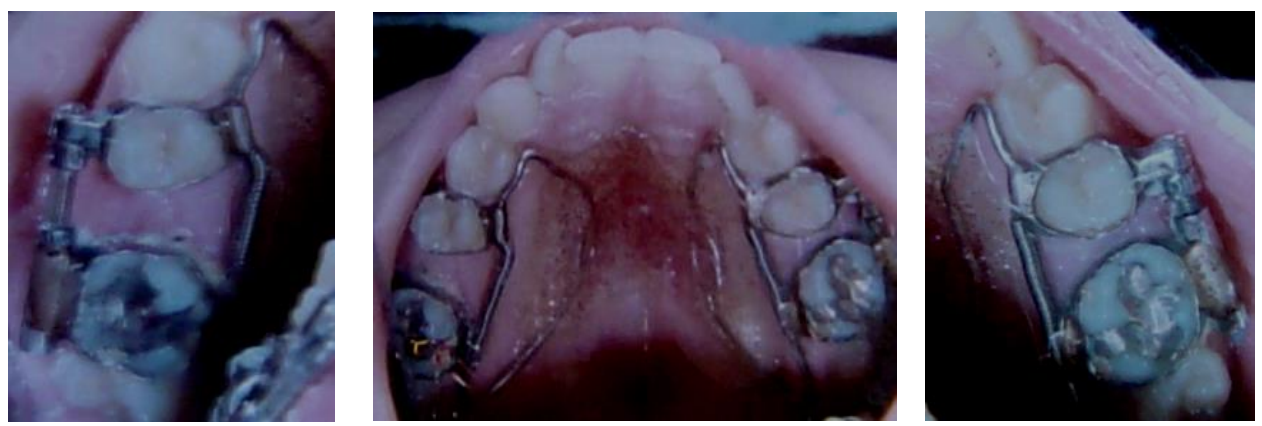

b. Post-distalization

Figure 2: The First Class appliance.

Volume 28 - December 2005 
The patients were seen at regular visits (every three weeks). During these visits the appliances were checked for any breakage. The First Class appliance was activated by the patient one quarter turn every day. On the other hand the Distal Jet was activated on the dental chair by the orthodontist. ${ }^{20}$ The disalization of the first molars were stopped when Class I molar relations were obtained.

For all patients plaster casts and panoramic radiographs were taken before distalization in addition to pre and post-distalization lateral cephalometric radiographs.

The cephalometric films were traced and the landmarks and reference points were located. ${ }^{25}$ Among these points were N, S, A, B, Me, Or, Po, Go and "mc" which is the maxillary first molar centroid., 25 Then the routine cephalometric lines and planes were made such as SN, NA, NB, FH, Mp, long axis of the maxillary central incisor, modified Frankfort horizontal "FHm" and Frankfort horizontal perpendicular "FHp". ${ }^{25,26} \mathrm{FHm}$ was made by drawing an inferior angle of seven degrees to SN plane through point S. FHp was drawn perpendicular to FHm through point S (Figure 3). Finally the following linear and angular measurements were done:

- SNA angle.

- SNB angle.

- ANB angle.

- FMA angle.

- SN-Mp angle.

- mc-FHp (mm.).

- 6-FHm (angle). The intrusection of perpendicular line from "mc" and "FHm".

- $\underline{1}$-NA angle.

- 1 -NA mm.

- 1 -SN angle.

Statistical analysis of the collected data was done using Microsoft Excel program on a personal computer. The means and standard deviations for the pre and post-distalization measurements and the changes were calculated for each group. Student test at 0.05 level of significance was used to evaluate the significance of differences between pre and post-distalization measurements for each group. Also, it was utilized to compare between the two groups. 
Egyptian

Orthodontic Journal

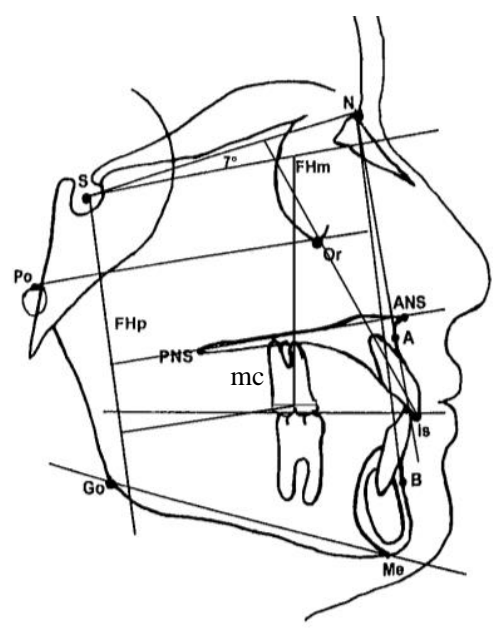

Figure 3: Cephalometric measurements.

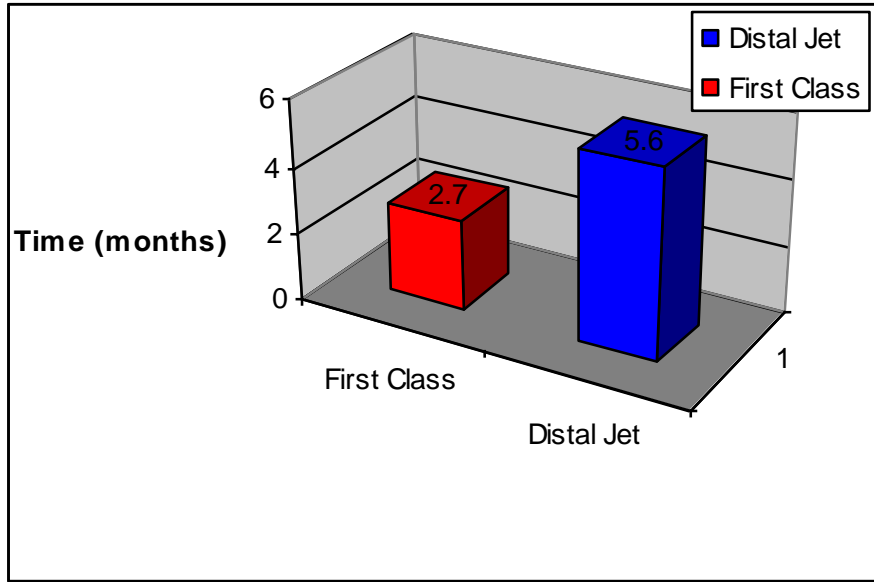

Figure 4: Distalization time of the Distal Jet and First Class appliances. 


\section{RESULTS}

The distalizing times of the Distal Jet and the First Class appliances are presented in table 1 and figure 4 . The First Class appliance had a significantly distalizing time than the Disal Jet.

Table $2 \& 3$ show the pre and post-distalization changes of the Distal Jet and the First Class appliances. Either appliance significantly distalized the maxillary first molars. However, the incisors were significantly tipped labially. Also, in both groups no significant skeletal changes in the anteroposterior direction were found. The mandible showed significant clock-wise rotation.

The comparison between the changes of the Distal Jet and the First Class appliances is illustrated in table 4 . The first molars significantly tipped distally in the First Class group than in the Distal Jet group. The other parameters were not significantly different.

Table 1: The means and standard deviations of distalization time of the Distal Jet and the First Class appliances and the result of $t$ test.

\begin{tabular}{|c|c|c|c|}
\hline & Distal Jet & First Class & P \\
\hline Time in months & $5.6 \pm 2.4$ & $2.7 \pm 1.6$ & 0.0004 \\
\hline
\end{tabular}

Table 2: The means and standard deviations of the pre and post-distalization measurements of the Distal Jet appliance group and the result of $t$ tests.

\begin{tabular}{|c|c|c|c|c|}
\hline Measurements & Pre-distalization & Post-distalization & Changes & P \\
\hline SNA & $79.6 \pm 3.7$ & $79.8 \pm 3.1$ & $0.2 \pm 1.4$ & 0.34 \\
\hline SNB & $77.2 \pm 2.7$ & $76.9 \pm 3.6$ & $-0.3 \pm 1.1$ & 0.42 \\
\hline FMA & $26.7 \pm 3.9$ & $27.8 \pm 4.2$ & $1.1 \pm 2.1$ & 0.04 \\
\hline SN-Mp & $38.1 \pm 4.5$ & $39.4 \pm 4.8$ & $1.3 \pm 1.9$ & 0.03 \\
\hline mc-FHp & $34.5 \pm 5.5$ & $30.9 \pm 6.1$ & $-3.6 \pm 1.2$ & 0.002 \\
\hline$\underline{6}$-FHm & $86.1 \pm 4.2$ & $83.3 \pm 5.1$ & $-2.8 \pm 3.9$ & 0.012 \\
\hline 1-NA & $23.3 \pm 5.6$ & $24.5 \pm 5.7$ & $1.2 \pm 2.8$ & 0.029 \\
\hline 1-NA & $4.7 \pm 4.3$ & $6.1 \pm 3.9$ & $1.4 \pm 1.7$ & 0.017 \\
\hline 1-SN & $103.3 \pm 5.1$ & $105.7 \pm 4.3$ & $2.4 \pm 2.5$ & 0.005 \\
\hline
\end{tabular}

Volume 28 - December 2005 
Table 3: The means and standard deviations of the pre and post-distalization measurements of the First Class appliance group and the result of $t$ tests.

\begin{tabular}{|c|c|c|c|c|}
\hline Measurements & Pre-distalization & Post-distalization & Changes & P \\
\hline SNA & $80.1 \pm 2.9$ & $80.2 \pm 3.2$ & $0.1 \pm 0.9$ & 0.47 \\
\hline SNB & $77.5 \pm 3.6$ & $77.1 \pm 2.3$ & $-0.4 \pm 1.4$ & 0.39 \\
\hline FMA & $25.6 \pm 5.1$ & $27.1 \pm 4.6$ & $1.5 \pm 1.1$ & 0.024 \\
\hline SN-Mp & $38.7 \pm 5.6$ & $40.2 \pm 4.3$ & $1.5 \pm 2.31$ & 0.038 \\
\hline mc-FHp & $35.3 \pm 4.7$ & $31.2 \pm 5.3$ & $-4.1 \pm 1.3$ & 0.002 \\
\hline$\underline{6}-\mathrm{FHm}$ & $86.2 \pm 5.1$ & $82.7 \pm 4.4$ & $-3.5 \pm 2.6$ & 0.023 \\
\hline$\underline{1}-\mathrm{NA}$ & $22.9 \pm 4.8$ & $24 \pm 5.1$ & $1.1 \pm 1.7$ & 0.032 \\
\hline$\underline{1}-\mathrm{NA}$ & $4.5 \pm 3.7$ & $5.7 \pm 4.1$ & $1.2 \pm 1.2$ & 0.041 \\
\hline 1-SN & $102.1 \pm 5.1$ & $103.5 \pm 4.8$ & $1.4 \pm 1.6$ & 0.02 \\
\hline
\end{tabular}

Table 4: The means and standard deviations of the post-distalization measurements changes of the Distal Jet and First Class appliances groups and the result of $t$ tests.

\begin{tabular}{|c|c|c|c|}
\hline Measurements & Distal Jet & First Class & P \\
\hline SNA & $0.2 \pm 1.4$ & $0.1 \pm 0.9$ & 0.63 \\
\hline SNB & $-0.3 \pm 1.1$ & $-0.4 \pm 1.4$ & 0.721 \\
\hline FMA & $1.1 \pm 2.1$ & $1.5 \pm 1.1$ & 0.573 \\
\hline SN-Mp & $1.3 \pm 1.9$ & $1.5 \pm 2.31$ & 0.682 \\
\hline mc-FHp & $-3.6 \pm 1.2$ & $-4.1 \pm 1.3$ & 0.04 \\
\hline$\underline{6}-\mathrm{FHm}$ & $-2.8 \pm 3.9$ & $-3.5 \pm 2.6$ & 0.05 \\
\hline$\underline{1-N A}$ & $1.2 \pm 2.8$ & $1.1 \pm 1.7$ & 0.089 \\
\hline$\underline{1-N A}$ & $1.4 \pm 1.7$ & $1.2 \pm 1.2$ & 0.133 \\
\hline$\underline{1-S N}$ & $2.4 \pm 2.5$ & $1.4 \pm 1.6$ & 0.037 \\
\hline
\end{tabular}


Egyptian

Orthodontic Journal

\section{DISCUSSION}

The results of the present study revealed that after 5.6 months the Distal Jet significantly moved the crowns of the maxillary first molars distally $3.6 \mathrm{~mm}$. The molars also tipped distally $2.8^{0}$. Those distal movements were accompanied with some anchorage loss as the maxillary incisors significantly tipped labially (table 2). This finding was matched with those of Bolla et al ${ }^{2}$, Abd El-Magid ${ }^{19}$ and Carano and $\mathrm{Testa}^{20}$. Slight but significant clock-wise mandibular rotation occurred as the both the FMA and SN-Mp angles were significantly increased. These results were in agreement with those of Abd El-Magid ${ }^{19}$ but were in disagreement with those of Bolla et $\mathrm{al}^{2}$. However, the mandibular rotation that occurred could be attributed to the presence of the maxillary and mandibular dentition in a triangular or wedge form. Backward movements of the molars to the tip of the wedge would open the anterior dentition. ${ }^{2}$ This finding support the hypothesis that distalization is not recommended for hyperdivergent patients. ${ }^{2,3}$ The latter results were in agreement with those of Abd El-Magid ${ }^{19}$ but were in disagreement with those of Bolla et $\mathrm{al}^{2}$. Finally, the Distal Jet had no significant skeletal effect in the anteroposterior direction hence no significant changes were found in SNA and SNB angles. Those effects were in harmony with those of other studies on the Distal Jet. ${ }^{2,19,20}$

Regarding the First Class appliance, the results of the present study showed that it distalized the maxillary first molars to Class I molar relation in $2.7 \pm 1.6$ months. The molars moved distally $4 \mathrm{~mm}$ with $3.5^{0}$ distal tipping. The tipping found was greater than those reported by Fortini et $\mathrm{al}^{22}$ and $\mathrm{Hafez}^{23}$. This could be explained by the difference in the sample size between the present study and those of Fortini et $\mathrm{al}^{22}$ and $\mathrm{Hafez}^{23}$. Anchorage loss was also found as the maxillary incisors were significantly flared labially. This was in agreement with those of $\mathrm{Hafez}^{23}$. Clock-wise mandibular rotation was also monitored with the First Class appliance. The FMA angle increased $1.5^{\circ}$. A similar increase was found in other study. ${ }^{23}$ Mandibular rotation could be due to the distal movements and tipping of the maxillary molars. ${ }^{2}$ The skeletal changes of the First Class appliance in the anteroposterior direction was not significant.

Comparison between the effects of the Distal Jet and the First Class appliances revealed that two main differences were found. The distalizing time of the First Class (2.7 months) was significantly shorter that that of the Distal Jet (5.6 months). The large distalizing force from the buccal and the palatal aspects of the molars utilized in the First Class appliance in comparison to the only palatal force used in the Distal Jet could be the cause. Reducing the distalizing time will subsequently decrease the treatment time and cost. On the other hand, 
the Distal Jet produced lesser molar tipping $\left(2.8^{0}\right)$ than the First Class appliance $\left(3.5^{0}\right)$. This could be attributed to the direction of distalizing forces in either appliance. The more the direction of force near the center of resistance of the molar which is located at the triforcation of the roots the more the bodily movement of the molar. In the Distal Jet the force passes near the center of resistance of the molar while in the First Class the force is more coronal.

In the present study though the First Class appliance utilized the second premolars as anchorage and the Distal Jet used the first premolars as anchorage there was no significant anchorage loss found between the two appliances. This result was matched with that of another investigators. ${ }^{2,19}$ Therefore in situations where the second premolars are not erupted, palatally positioned or impacted and it is difficult to use them as anchorage units, the first premolars could be utilized as anchorage units.

According to the results of the present study the following could be concluded; both the Distal Jet and the First Class appliance were effective and reliable methods for maxillary first molars distalization. The First Class distalized the maxillary molars in shorter time than the Distal Jet but with greater distal tipping. Labial tipping of the incisors and clock-wise mandibular rotation occurred with both appliances. No significant skeletal changes in the anteroposterior direction found with either appliances.

\section{REFERENCES}

1. Germane N, Líndauer S J, Rubenstein $L \mathbf{K}$, Revere $\mathbf{J} \mathbf{H}$, and Isaacson $\mathbf{R} \mathbf{J}$ : Increase in arch perimeter due to orthodontic expansion. Am J Orthod Dentofacial Orthop, 1991; 100, 421-427.

2. Bolla E, Muratore F, Carano A and Bowman J: Evaluation of Maxillary Molar Distalization With the Distal Jet: A Comparison With Other Contemporary Methods. Angle Orthod J, 2002; 72: 481-494.

3. Bowman S J: Class II combination therapy. J Clin Orthod. 1998; 32:611-620.

4. Cetlin N M and Ten-Hoeve A: Nonextraction treatment. J Clin Orthod, 1983; 17:396-413.

5. Sahm G, Bartsch A and Witt E: Reliability of patient reports on compliance. Eur J Orthod, 1990;12:438-446.

6. Nanda R S and Kieri M J: Prediction of cooperation in orthodontic treatment. Am J Orthod Dentofacial Orthop, 1992; 102:15-21. 
7. Cureton S L, Regennitter $\mathbf{F} \mathbf{J}$ and Yancy $\mathbf{J}$ M: Clinical versus quantitative assessment of headgear compliance. Am J Orthod Dentofacial Orthop, 1993; 104:277-284.

8. Muse D S, Fillman M J, Emmerson W J and Mitchell R D: Molar and incisor changes with the Wilson rapid molar distalization. Am J Orthod Dentofacial Orthop, 1993; 103:556-565.

9. Jones R D and White M J: Rapid Class II molar correction with an open-coil jig. J Clin Orthod, 1992; 26:661-664.

10. Brickman C D, Sinha $\mathbf{P} \mathbf{K}$ and Nanda R S: Evaluation of the Jones jig appliance for distal molar movement. Am J Orthod Dentofacial Orthop, 2000; 118:526-534.

11. Haydar S and Uner O: Comparison of Jones jig molar distalization appliance with extraoral traction. Am J Orthod Dentofacial Orthop, 2000; 117:49-53.

12. Ghosh J and Nanda R S: Evaluation of an intraoral maxillary molar distalization technique. Am J Orthod Dentofacial Orthop, 1996; 110:639-646.

13. Chaqués-Asensi $\mathbf{J}$ and Kalra V: Effects of the pendulum appliance on the dentofacial complex. J Clin Orthod, 2001; 35:254-257.

14. Byloff F K and Darendeliler M A: Distal molar movement using the pendulum appliance. Part 1: clinical and radiological evaluation. Angle Orthod, 1997; 67:4249-260.

15. Bussick $\mathbf{T} \mathbf{J}$ and McNamara $\mathbf{J} \mathbf{A}$ : Dentoalveolar and skeletal changes associated with the pendulum appliance. Am J Orthod Dentofacial Orthop, 2000; 117:333343.

16. Kinzinger G S, Fritz U B, Sander F G and Diedrich P R: Efficiency of a pendulum appliance for molar distalization related to second and third molar eruption stage. Am J Orthod Dentofacial Orthop, 2004;125:8-23.

17. Gianelly A A, Vaitas A S, Thomas W M and Berger D G: Distalization of molars with repelling magnets. J Clin Orthod, 1988; 22:40-44.

18. Gianelly A A, Vaitas, A S and Thomas W M: The use of magnets to move molars distally, Am J Orthod, 1989: 96:161-167.

19. Abd El-Magid M T: Evaluation of the Distal Jet and Multi-Distalizing arch in distalization of maxillary molars. MD Thesis. Orthodontic Department, Mansoura University, 2003 
Egyptian

Orthodontic Journal

20. Carano A and Testa M: The Distal Jet for upper molar distalization. J Clin Orthod, 1996; 30,374-380.

21. Patel A: Analysis of the Distal Jet Appliance for Maxillary Molar Distalization. [unpublished master's thesis] Oklahoma City: Department of Orthodontics, University of Oklahoma; 1999. Quted from: Bolla E, Muratore F, Carano A and Bowman J: Evaluation of Maxillary Molar Distalization With the Distal Jet: A Comparison With Other Contemporary Methods. Angle Orthod J, 2002; 72: 481-494.

22. Fortini A, Lupoli M and Parri M: The First Class appliance for rapid molar distalization. J Clin Orthod, 1999; 33: 322-328.

23. Hafez A M: Distalizing the maxillary permanent molars using the First Class appliance. MD Thesis. Orthodontic Department, Mansoura University, 2004.

24. El-kadi A, Labib A and Ramadan A: Cephalometric evaluation for First Class molar distalizer. Egyp Dent J, 2001; 47:615-621.

25. Rakosi T: An atlas and manal of cephalometric radiology. Wolfe Medical Publication. Ltd. 1982.

26. Sidhu M S and Kharbanda P: Cephalometric analysis of changes produced by modified Herbst appliance in the treatment of Class II division 1 malocclusion. Br J Orthod, 1995; 22: 1-12. 